\title{
Game Dynamics and Bounded Rationality
}

\author{
Hans W. Gottinger \\ Stratec Munich Germany
}

\begin{abstract}
Suppose a game is played repeatedly by a finite collection of players. At every step, each player plays his optimal strategy given the observed probabilities of play for the strategies used by the other players. This generates a (time-dependent) map of the joint strategy space into itself known as 'fictitious play'(FP). This map can be approximated by a discontinuous vector field. 'Weak' solutions for this dynamics are defined, and shown to exist and be unique under certain generic conditions. These weak solutions are also shown to be limits of the original discrete dynamics as the step size approaches zero. It is shown that this process lends itself to a reasonable interpretation of bounded rationality in the appropriate context.
\end{abstract}

Keywords: Games, Dynamic Systems, Complexity, Bounded Rationality.

\section{INTRODUCTION}

By embedding game theory in a dynamical context, one may hope to find a basis of attraction for various Nash equilibria, thereby interpreting the choices of a particular equilibrium as a consequence of the boundary conditions of the dynamical game. Further, a complicated dynamics which fails to converge to a Nash equilibrium may indicate a fundamental inconsistency in the game or a breakdown of von Neumann rationality. Beyond the technical results proposed below the linkage between fictitious play games and dynamical systems suggests on some scale interesting features of social and economic dynamics toward equilibria states.

Within different dynamical contexts, the same game may display very different features. A given dynamics provides its own notion of a solution concept, one which may be inherently limited by the possibility of exotic behaviour.

An example of embedding a game in a dynamical context is the game of fictitious play, as well as similar cases of evolutionary type games (Binmore, 1987).

Consider the repeated play of a game $G$. At each stage, there are empirically observed mixed strategies for each player given by $x_{j}{ }^{(i)}(t)$ (number of times the i-th player has used strategy $j$ ). Now suppose that each player plays his optimal strategy given the currently observed mixed strategies $x^{(1)}(t) \ldots, X^{(\mathrm{n})}(t)$. Since the payoffs are multilinear functions of the observed mixed strategies, the optimal strategy will be a pure strategy. (Ties can be broken arbitrarily.) This generates a discrete-time dynamics on strategy space given by

$$
\left.x_{j}^{(i)}(t+1)=t x_{j}^{(i)}(t)+\Gamma_{j}^{(i)}(x)(t)\right) / t+1 .
$$

where $\Gamma_{j}^{(i)}(x)=1$ if the optimal strategy for the i-th player given the joint mixed strategy choice $x$ is $j$, and 0 otherwise.

The simplicity of this dynamics allows a crucial simplification. Instead of working with all possible histories of strategies, we need only consider average tendencies to use strategies. 
Instead of an unwieldy discrete strategy space, we have a convenient continuous strategy space. In effect, the decision to throw out detailed knowledge of the particular order in which strategies are played in favour of overall tendencies is a kind of bounded rationality, where the players use simple playing habits to confront potentially complex situations (Megiddo, 1986, Kalai, 1988), see also Gottinger(1990).

Brown (1951) and Robinson (1951) introduced this 'fictitious play' dynamics and were able to prove it converges to the von Neumann equilibrium for the two-player, zero-sum case. Later Shapley (1964) gave an example where fictitious play leads to oscillatory dynamics. In the context of bounded rationality fictitious play is mentioned by Kreps (1990) and further pursued by Fudenberg and Kreps (1993).

Later on, there has been an overwhelming interest in fictitious-play dynamics (FPD).

We study a continuous-time analogue of fictitious play by using concepts and tools of dynamical systems (Smale, 1980a). Alternative models of continuous fictitious play (CFP) have been proposed by Gaunersdorfer and Hofbauer (1995) and Monderer et al. (1996).

In Gaunersdorfer and Hofbauer (1995), existence can be settled by general results from the theory of differential inclusions. Uniqueness is discussed in Hofbauer (1995) where in particular examples of non-uniquess are discussed. CFP has been initialized earlier by Rosenmüller (1971), but convergence results of 2x2 games using best-response dynamics has only later been proved by Metrick and Polak (1994). It can also be mentioned that, except for a scaling of time (which does not affect the orbits), CFP is equivalent to the best response dynamics (Matsui, 1992). We obtain an approximation result for CFP by the original discrete fictitious play (DFP) with small step size. We show that if the trajectory crosses the hyperplanes which separate best response regions only a finite number of times, DFP approximates CFP. The result covers the cases (a) for a bounded interval of time, or (b) convergence to a pure strategy equilibrium. Another interesting problem concerns the general behaviour of DFP and CFP, allowing convergence to mixed strategy equilibrium (Metrick and Polak, 1994) but this issue is not further resolved.

The model of FP, described in Section 2, is an appealing and simple embodiment of a process in which boundedly rational players react to each other's play, creating a dynamical system with the state space being the set of mixed strategy profiles. A difficulty with the model is that its associated gradient field is discontinuous at strategy profiles on boundaries between different best response regions. Because of this, standard existence and uniqueness theorems for dynamic systems do not apply.

The paper addresses this gap. Essentially, we define a non-degeneracy condition so that the dynamical system passes through the (typically lower dimension) regions of discontinuity rather than travelling within one of these troubling regions. In Section 3 we show (Theorem 3.3 ) that a sufficient condition for this non-degeneracy in 2 player games is that for each pure strategy for player 2, player 1 is not indifferent between any two of his pure strategies, and vice versa. So for 2 person games 'mostly' this non-degeneracy is satisfied. The core of the paper in Section 4 are Theorems 4.1 and 4.2. Theorem 4.1 says that as long as the path avoids points of degeneracy, it is unique. Theorem 4.2 says that solutions exist.

These theorems are for continuous time, in Section 5 conditions are established under which the discrete time solution approximates the continuous time solution as the step size grows smaller. 
In Section 6, a specific FP dynamics is given, and vector fields corresponding to two strategy vs. two strategy games are classified. Section 7 concludes the paper.

\section{THE DISCRETE FICTITIOUS PLAY MODEL}

Following Robinson (1951) let us formalize the notion of discrete fictitious play. For our purposes, we can take a restricted notion of a 'game'. ${ }^{1}$

Definition. A game $G$ is a triple $(N, \hat{S}, \hat{P})$ where $N \in Z^{+}$is a positive integer, $\hat{S}=\left(\hat{S}^{(1)}, \ldots, \hat{S}^{(N)}\right)$ is an $N$ tuple of finite sets $\hat{S}^{(i)}=\left\{e_{1}^{(i)}, \ldots, e_{n}^{(i)}\right\}$ and $\hat{P}=\left(\hat{P}^{(1)}, \ldots, \hat{P}^{(N)}\right)$ is an N-tuple of maps

$$
\hat{P}(i): \hat{S}^{(1)} x . . x \hat{S}(N) \rightarrow \Re .
$$

Here $N$ is the number of players, $\hat{S}^{(i)}$ is the collection of strategies for the $\mathrm{i}$-th player, $\hat{P}(i)$ is the payoff for the $i$-th player, and $\mathfrak{R}$ the real line. assignment of a non-negative probability $x_{j}^{(i)}$ to each possible 'pure' strategy $e_{j}^{(i)} \in \hat{S}^{(i)}$ in the original game.

Definition. A strategy space $S^{(i)}$ corresponding to the ordered finite set of strategies $\hat{S}^{(\mathrm{i})}=\left\{e_{1}(1), \ldots, e_{n}{ }^{(i)}\right\}$ for the $\mathrm{i}$-th player is the set of convex formal sums

A mixed strategy is just an

$$
S=\left\{\sum_{j=1}^{n_{i}} x_{j}^{(i)} e_{j}^{(i)} \mid \sum_{j=1}^{n_{i}} x_{j}^{(i)}=1, x_{j} \geq 0\right\}
$$

We identify it with the $\left(n_{i}-1\right)$ dimensional simplex $S^{n_{i}^{-1}} \subset \mathfrak{R}_{i}^{n} \mathrm{~S}^{\mathrm{n}_{\mathrm{i}}^{-1}} \subset \mathfrak{R}_{\mathrm{i}}^{\mathrm{n}}$ by

$$
\begin{gathered}
S^{(i)} \rightarrow S^{n_{i}-1} \subset \Re_{i}^{n}, \\
\sum_{j=1}^{n_{i}} x_{j}^{(i)} e_{j}^{(i)} \rightarrow\left(x_{1}^{(i)}, \ldots, x_{n_{i}}^{(i)}\right) .
\end{gathered}
$$

Under this identification, we have

$$
e_{j}^{(i)} \rightarrow e_{j} \in \Re^{n_{i}}
$$

where $e_{j}$ is just the $\mathrm{j}$-th standard basis vector in $\mathfrak{R}^{n_{t}}$. The strategy space $S$ of a game $G=(N, \hat{S}, \hat{P})$ is

$$
S=S^{(1)} x \ldots x S^{(M)} \subset \Re^{n_{1}} x \ldots x \Re^{n_{N}}
$$

As a notational convention, we write an element $x \in S$ as

$$
\begin{gathered}
x=\left(x^{(1)}, \ldots, X^{(N)}\right), \\
x^{(i)}=\left(x_{1}^{(i)}, \ldots, x_{n_{i}}^{(i)}\right), \quad i=1, \ldots, N .
\end{gathered}
$$


Definition. The payoff to the $i$-th player for a given point $x$ in strategy space is:

$$
P^{(i)}\left(x^{(1)}, \ldots, x^{(N)}\right)=\sum_{\alpha_{1}=1}^{n_{i}} \ldots \sum_{\alpha_{N}=1}^{n_{N}} x_{\alpha 1}^{(1)} \ldots x_{\alpha N}^{(N)} \hat{P}^{(i)}\left(e_{\alpha 1}^{(1)}, \ldots, e_{\alpha N}^{(N)}\right)
$$

The payoff is clearly a multilinear function of the $N$ arguments $x^{(1)}, \ldots, X^{(N)}$.

The payoff to the $i$-th player for using the j-th strategy is:

$$
E_{j}^{(i)}(X)=P^{(i)}\left(X^{1}, \ldots, X^{(i-1)}, e_{j}^{(i)}, X^{(i+1)}, \ldots, X^{(N)}\right)
$$

This payoff is independent of $x^{(i)}$ itself.

Definition. The optimal strategy set for the i-th player is the set of pure strategies with maximum payoff:

$$
O P T^{(i)}(x)=\left\{e_{\alpha^{(i)}} \mid \max _{\beta} E_{\beta}^{(i)}(x)=\mathrm{E}_{\alpha}^{(i)}(x)\right\} .
$$

Robinson left the choice of strategy arbitrary in the case of ties. We specify it for the sake of concreteness by choosing the optimal strategy with the smallest index.

Definition. The optimal strategy for the discrete dynamics is

$$
\begin{gathered}
\Gamma^{(i)}(x)=e_{\alpha}^{(i)}, \\
\alpha=\min \left\{\beta \mid e_{\beta}^{(i)} \in O P T^{(i)}(x)\right\} .
\end{gathered}
$$

With these definitions in place, we can finally discuss the fictitious play dynamics.

Definition. The discrete fictitious play dynamics with step size $h>0$ associated with a game $G=$ $(N, \hat{S}, \hat{P})$ is the map:

$$
\begin{gathered}
\psi: S x T \rightarrow S x T, \\
(x, t) \rightarrow(t x+h \Gamma(x) / t+h, t+h),
\end{gathered}
$$

where $T=\left\{n h \mid n \geq n_{0} \geq 0\right\}$. The dynamics also require an initial condition $\left(x, t_{0}\right), t_{0}=n_{0} h$.

The dynamics is clearly well defined for all $t \in T$. The dynamics is $1-1$ but not onto. The first component $\psi$ represents a running time average of the strategy choices of the players; it is a vector of empirically observed probabilities for the various strategies of each player. The following result is immediate:

Theorem 2.1. Suppose $\Gamma(\psi(t))$ remains constant for $\tau_{0} \leq t \leq \tau_{1}=\tau_{0}+n h$.

Then

$$
\Psi\left(\tau_{1}\right)=\tau_{0} \Psi\left(\tau_{0}\right)+\left(\tau_{1}-\tau_{0}\right) \Gamma\left(\Psi\left(\tau_{0}\right)\right) / \tau_{1}
$$

\section{THE CONTINUOUS FICTITIOUS PLAY MODEL}

We now introduce a continuous-time version of the fictitious play dynamics (Robinson discusses only the discrete $h=1$ case). Letting $h \rightarrow 0$ in (2.1), we obtain:

$$
\lim _{h \rightarrow 0} \psi(x, t)-(x, t) / h=\lim _{h \rightarrow 0}(t x+h \Gamma(x) / h(t+h)-x / h, 1)=(\Gamma(x)-x / t, 1) .
$$


Consider the flow defined by:

$$
d x / d t=\Gamma(x)-x / t, t \in\left[t_{0}, t_{1}\right), t_{0}>0 .
$$

Whenever $O P T^{(i)}(x)$ contains two or more points there is a potential discontinuity in the i-th component of the vector field. For such $x$, there is no reason for the i-th player to prefer one optimal strategy over another. We can define a (discontinuous) vector field by leaving the vector field undefined on the ambiguous set. Since traditional existence-uniqueness theorems do not apply to such situations, we will have to build some new ones, starting from a notion of "weak solution" of the vector field.

Definition. The region of discontinuity for the i-th player is

$$
D^{(i)}=\left\{x \mid \operatorname{card}\left(O P T^{(i)}(x)\right) \geq 2\right\}
$$

It is the set of points where the $\mathrm{i}$-th player has an indeterminate choice of optimal strategy. Since $\operatorname{OPT}^{(i)}(x)$ is independent of $\mathrm{x}^{(i)}$, we have

$D^{(i)} \approx \mathrm{S}^{(i)} \times F$,

$F \subset S^{(1)} \times \ldots S^{(i-1)} \times S^{(i+1)} \times \ldots S^{(N)}$,

where the homeomorphism involves a simple relabelling of players. Generically, $D^{(i)}$ is of codimension 1 in $S$.

Theorem 3.1. $D^{(i)}$ is closed for $i=1, \ldots, N$.

Proof. From the definition of $O P T^{(i)}(\mathrm{x})$, we have

$$
D^{(i)}=\bigcup_{1 \leq j<k \leq n_{i}}\left\{x \mid E_{j}^{(i)}(x)-E_{k}^{(i)}(x)=0\right\}
$$

Let $f_{j k}(x)=E_{j}^{(i)}(x)-E_{k}{ }^{(i)}(x)$. Then $D^{(i)}$ is the finite union of $f_{j k}{ }^{1}(0)$. Since each $f_{j k}$ is continuous, in fact a multilinear function of $x^{(1)}, \ldots, X^{(i-1)}, X^{(i+1)}, \ldots X^{(N)}$, each $f_{j k}{ }^{1}(0)$ is closed. Thus $D^{(i)}$ is the finite union of closed sets and is itself closed.

For later use, we will need the following two definitions.

Definition. For $n \geq 2$, we define the region of $n$-fold intersection as

Clearly, $D_{2}=\cup^{N_{\mathrm{j}=1}} D^{(i)}$.

$$
D_{n}=\left\{x \mid \exists i \operatorname{card}\left(O P T^{(i)}(x)\right) \geq n\right\} .
$$

Definition. The region of full discontinuity is

$$
D_{22}=\bigcup_{1 \leq i<j \leq N} D^{(i)} \cap D^{(i)}
$$

This is a set of points where there are simultaneously ambiguous optimal choices for two or more players. Generically, $D_{22}$ is of codimension 2 in $S$ (being a finite union of intersections of two sets of codimension 1 ).

We can now define the vector field. 
Definition. The continuous fictitious play dynamics is defined by

$$
\frac{d x^{(i)}}{d t}=\left\{\frac{\Gamma^{(i)}(x)-x^{(i)}}{t} \text { if } x \in S \backslash D^{(i)}\right. \text {; }
$$

$t \in\left[t_{0}, t_{1}\right), t_{0}>0$

Since the vector field is not everywhere defined, we cannot demand of a solution that it can be everywhere differentiable. However, we can demand that it be differentiable where defined and continuous elsewhere. For the definition to be useful, it must also treat an exceptional case (the region of full discontinuity) carefully.

Definition. A weak solution to (3.1) is a map

$$
\phi:\left[t_{0}, t_{1}\right) \rightarrow S \subset \Re^{n_{1}} x \ldots x \Re^{n_{N}}
$$

which satisfies:

(1) $\phi$ is continuous.

(2) $d \phi^{(i)} / d t=\Gamma^{(i)}(\phi(t))-\phi^{(i)}(t) / t$ for $\phi(t) \in S \backslash\left(D^{(i)} \cup D_{22}\right)$.

(3) $\phi(\tau) \in D_{22} \rightarrow \phi(t)-\phi(\tau)$ for $t>\tau$.

Condition (3) is chosen to handle the anomalous condition of full discontinuity. It allows Nash equilibria to be dynamical equilibria, as we shall see in Section 6, (Recently, related conditions for slightly different processes have been suggested by Hofbauer (1995) and Monderer et al (1996)).

Theorem 3.2. Suppose $\phi(t) \in S \backslash D_{2}$ for $\tau_{0} \leq t \leq \tau_{1}$. Then

$$
\phi\left(\tau_{1}\right)=\tau_{0} \phi\left(\tau_{0}\right)+\left(\tau_{1}-\tau_{0}\right) \Gamma\left(\phi\left(\tau_{0}\right)\right) / \tau_{1}
$$

Proof. Straightforward integration of the vector field, using the constancy of $\Gamma(\phi(t))$.

Remarkably, the result is the same as for the discrete dynamics (2.1) which is the basis of similar results by Monderer et al (1996).

The following two examples illustrate two different kinds of pathological dynamics.

Example 1. Consider a two player game where each player has two strategies $\left(N=2, n_{1}=n_{2}=\right.$ 2). Let

$$
P^{(1)}=\left(\begin{array}{ll}
0 & 0 \\
0 & 0
\end{array}\right), \quad P^{(2)}=\left(\begin{array}{ll}
0 & 0 \\
0 & 0
\end{array}\right)
$$

Then $D^{(1)}=D^{(2)}=S$. Hence for any starting $\phi\left(t_{0}\right) \in S$, we have $\phi\left(t_{0}\right) \in D_{22}$, and we obtain the unique (trivial) solution $\phi(t)=\phi\left(t_{0}\right)$ for $t \in\left[t_{0}, t_{1}\right)$.

Example 2. Now consider the two player two strategy game with

$$
P^{(1)}=\left(\begin{array}{ll}
1 & 0 \\
0 & 0
\end{array}\right), \quad P^{(2)}=\left(\begin{array}{ll}
0 & 1 \\
0 & 1
\end{array}\right) .
$$


Then $D^{(1)}=\left\{x \mid x_{1}(2)=0\right\}, D^{(2)}=\emptyset, D_{22}=D^{(1)} \cap D^{(2)}=\emptyset$. Since $\Gamma^{(2)}(x)=e_{2}(2)$ for $\forall x \in S$, we have $d \phi_{1}{ }^{(2)} / d t=-\phi_{1}{ }^{(2)} / t=\forall t \in\left[t_{0}, t_{1}\right)$. If $\phi_{1}{ }^{(2)}\left(t_{0}\right)=0$, then $\phi_{2}{ }^{(2)}(t)=0, \forall t \in\left[t_{0}, t_{1}\right)$. Since $\phi(t) \in D^{(1)} \forall t$, we see that condition (2) of

equation (3.1) is never satisfied. So $\left(\phi^{(1)}(t), e_{2}^{(2)}\right)$ is a weak solution for any continuous map $\phi^{(1)}:\left[t_{0}, t_{1}\right) \rightarrow S^{(1)}$.

To avoid the extreme non-uniqueness evidenced by the second example, we need to impose a non-degeneracy condition on games. Suppose that $x^{(i)} \in D^{(i)}$. We need to ensure that the movement of the remaining $N-1$ players will pull the i-th player away from the region of discontinuity $D^{(i)}$. We are led to the following definition.

Definition. The degenerate set $Z$ for a game $G$ is the set of points $x \in S$ satisfying these two conditions:

(1) $x \in D^{(\mathrm{i})} \backslash D_{22}$.

(2) We have $O P T^{(\mathrm{i})}(x)=A \cup B$ with card $(A) \geq 2$ and $\exists \lambda_{0}>0$ such that

$\forall \lambda \in\left[0, \lambda_{0}\right] \forall e_{\alpha}^{(i)}, e_{\beta}{ }^{(i)} \in A, \alpha \neq \beta E_{\alpha}^{(i)}((1-\lambda) x+\lambda \Gamma(x))-E_{\beta}{ }^{(i)}((1-\lambda) x+\lambda \Gamma(x))=0$,

$\forall \lambda \in\left(0, \lambda_{0}\right] \forall e_{\alpha}{ }^{(i)} \in \mathrm{A}, e_{\beta}{ }^{(i)} \in B E_{\alpha}{ }^{(i)}((1-\lambda) x+\lambda \Gamma(x))-E_{\beta}{ }^{(i)}((1-\lambda) x+\lambda \Gamma(x))>0$

Condition (2) is precisely the condition for a solution to remain in $D^{(i)}$ for a whole interval of time.

Theorem 3.3. Let $(N, \hat{S}, \hat{P})$ be a two-player game. Then $Z$ is empty if

$$
\begin{aligned}
& P^{(1)}\left(e_{\alpha}{ }^{(1)}, e_{j}^{(2)}\right) \neq P^{(1)}\left(e_{\beta}^{(1)}, e_{j}^{(2)}\right) \text { for } 1 \leq j \leq n_{2}, 1 \leq \alpha<\beta \leq n_{1}, \\
& P^{(2)}\left(e_{j}(1), e_{\alpha}{ }^{(2)}\right) \neq P^{(2)}\left(e_{j}^{(1)}, e_{\beta}{ }^{(2)}\right) \text { for } 1 \leq j \leq n_{1}, 1 \leq \alpha<\beta \leq n_{2} .
\end{aligned}
$$

Proof. Suppose $x \in D^{(1)} \backslash D_{22}$ and $x \in Z$. By the definition of $Z$, we can find $1 \leq \alpha<\beta \leq n_{2}, \lambda>0$ with

(1) $E_{\alpha}^{(1)}(x)-E_{\alpha}^{(1)}(x)=0$.

(2) $E_{\alpha}^{(1)}((1-\lambda) x+\lambda \Gamma(x))-E_{\beta}^{(1)}((1-\lambda) x+\lambda \Gamma(x))=0$.

Set $\Gamma^{(2)}(x)=e_{j}^{(2)}$. Since $N=2, E_{k}^{(i)}$ is linear in $x$. Using (1), we see that (2) reduces to: (2') $P^{(1)}\left(e_{\alpha}^{(1)}, e_{j}^{(2)}\right)-P^{(1)}\left(e_{\beta}^{(1)}, e_{j}^{(2)}\right)=0$.

However, this contradicts our assumption. A similar contradiction occurs if $x \in D^{(2)} \backslash D_{22}$ and $x \in$ $Z$. Hence $Z=\emptyset$ as asserted.

The pathological dynamics for the second example above occur on the degenerate set, as can easily be checked.

\section{EXISTENCE AND UNIQUENESS THEOREMS}

We are now ready to prove something definitive about the uniqueness of weak solutions (in forward time).

Theorem 4.1. Uniqueness and Forward Time. Suppose that $\phi_{1}(t)$ is a weak solution of (3.1) defined on some interval $\left[t_{0}, t_{1}\right)$. Suppose that $\forall \mathrm{t} \in\left[t_{0}, t_{1}\right), \phi_{1}(t) \notin \mathrm{Z}$. Then any weak solution $\phi_{2}(t)$ of (3.1) defined on such that $\left[t_{0}, t_{1}\right)$. Such that $\phi_{1}\left(t_{0}\right)=\phi_{2}\left(\mathrm{t}_{0}\right)$ must satisfy $\phi_{1}(t)=\phi_{2}(t) \forall t \in$ $\left[t_{0}, t_{1}\right)$. 
Proof. Assume otherwise. Then $\exists t \in\left[t_{0}, t_{1}\right)$ such that $\phi_{1}(t) \neq \phi_{2}(t)$. Set

$$
\tau=\infty\left\{t \mid \phi_{1}(t) \neq \phi_{2}(t) \& \mathrm{t} \in\left[t_{0}, t_{1}\right)\right\}
$$

By the continuity of $\phi_{1}$ and $\phi_{2}$, we must have that $\phi_{1}(\tau)=\phi_{2}(\tau)$. Then one of the following must hold:

Case 1. $\phi_{1}(\tau) \notin D^{(i)}$ for $\mathrm{i}=1, \ldots, N$.

Then the vector field is $C^{1}$ in a neighbourhood of $\phi_{1}(t)$ for $t \in\left[\tau, \tau_{1}\right)$ for some $\tau_{1}>\tau$. By the local uniqueness theorem for smooth vector fields, $\phi_{1}(t)$ is unique on $\left[\tau, \tau_{1}\right)$. This contradicts the definition of $\tau$.

Case 2. $\phi_{1}(\tau) \in D_{22}$.

By condition (3) of the definition of a weak solution to (3.1), we must have ${ }_{1}(t)={ }_{2}(t)={ }_{1}(\tau)$ for $t$ $\in\left[\tau, \tau_{1}\right)$ for some $\tau_{1}>\tau$. This again contradicts the definition of $\tau$.

Case 3. $\phi_{1}(\tau) \in D^{(i)}$ for some $i$ and $\phi_{1}(\tau) \notin D^{(j)}$ for $j \neq i$.

Since $D^{(j)}$ is closed for each $j=1, \ldots, N$ and $\phi_{1}$ is continuous, we can find some $\tau_{1}>\tau$ so that

$$
\phi_{j}(t) \notin D^{(j)} \text { for } j \neq i, t \notin\left[\tau, \tau_{1}\right) .
$$

Hence, $\Gamma^{(j)}\left(\phi_{1}(t)\right)=\Gamma^{(j)}\left(\phi_{1}(\tau)\right)$ for $t \in\left[\tau, \tau_{1}\right)$. By theorem 3.2, we have

$\phi_{1}^{(j)}(t)=(1-\lambda(t)) \phi_{1}(j)(\tau)+\lambda(t) \Gamma^{(j)}\left(\phi_{1}(\tau)\right)$ for $j \neq i, t \in\left[\tau, \tau_{1}\right)$.

$\lambda(t)=t-\tau / t$

Since $E_{\alpha}{ }^{(i)}$ is independent of $x^{(i)}$, we have

$$
E_{\alpha}^{(i)}\left(\phi_{1}(t)\right)=E_{\alpha}{ }^{(i)}\left((1-\lambda(t)) \phi_{1}(\tau)+\lambda(t) \Gamma\left(\phi_{1}(\tau)\right)\right) \text { for } t \in\left[\tau, \tau_{1}\right)
$$

The condition that $\phi_{1}(\tau) \notin Z$ guarantees that

$\exists \tau_{2}, \tau<\tau_{2} \leq \tau_{1}, \exists \alpha \forall \beta$ with $E_{\alpha}{ }^{(i)}\left(\phi_{1}(t)\right)>E_{\beta}{ }^{(i)}\left(\phi_{1}(t)\right)$ for $\beta \neq \alpha, t \in\left[\tau, \tau_{2}\right)$.

So for $t \in\left(\tau, \tau_{2}\right)$ we have $\phi_{1}(t) \notin D_{2}$. Since $\phi_{1}(\tau)=\phi_{2}(\tau)$, we can apply the standard uniqueness result to obtain $\phi_{1}(t)=\phi_{2}(t)$ for $t \in\left[\tau, \tau_{2}\right)$. This contradicts the definition of $\tau$, so we are done.

To complement the uniqueness result, we have the following existence theorem.

Theorem 4.2. Existence in Forward Time. Suppose $G$ is a fixed game. Then $\forall x \in S, \forall t_{0}>0$ there is a weak solution defined on $\left[\tau_{0}, \infty\right)$ which satisfies the initial condition $\phi\left(t_{0}\right)=x$.

Proof. It is clear from the uniqueness theorem that if $\phi(\tau) \notin D_{22}$ it is possible to extend the weak solution $\phi$ to a solution on $\left[t_{0}, \tau /\right]$ with $\mathrm{r} />\tau$ and $\phi(\tau /) \in D_{2}$. If $\phi(\tau) \in D_{22}$ then we can take $\phi(t)=\phi(\tau)$ for $t \in[\tau, \infty)$.

One possibility remains. Suppose the extensions to larger $t$ are bounded above. Let the extensions be to $\left\{\tau_{n}\right\}_{\eta=1}{ }^{\infty}$. Let $\tau=\lim _{n \rightarrow \infty} \tau_{n}$. By the continuity of $\phi, \phi(\tau)=\lim _{n \rightarrow \infty} \phi\left(\tau_{n}\right)$. Since it is impossible to have infinitely many consecutive intersections with the same $D^{(i)}$, it must be possible to find $i \neq j$ and subsequences 


$$
\begin{aligned}
& \left\{\tau_{\alpha_{n}}\right\} \rightarrow \tau \text { with } \phi\left(\tau_{\alpha_{n}}\right) \in D^{(i)}, \\
& \left\{\tau_{\beta_{n}}\right\} \rightarrow \tau \text { with } \phi\left(\tau_{\beta_{n}}\right) \in D^{(i)},
\end{aligned}
$$

Since $D^{(i)}$ and $D^{(j)}$ are closed, we have that $\phi(\tau) \in D^{(i)}$ and $\phi(\tau) \in D^{(j)}$. Hence $\phi(\tau) \in D_{22}$, and we can take $\phi(t)=\phi(\tau)$ for $t \in[\tau, \infty)$, as before.

\section{APPROXIMATING THE CONTINUOUS DYNAMICS WITH THE DISCRETE DYNAMICS}

Despite the discontinuous nature of the vector field (3.1), it seems plausible that it can be approximated arbitrarily well as $h \rightarrow 0$ by the discrete fictitious play dynamics (2.1). For the approximation to go through, there must be only finitely many intersections of the continuous solutions with the regions of discontinuity $D^{(i)}$. In addition, each intersection must be generic, in a sense to be made precise below.

Notation. We shall write $\Psi_{h}(t)$ for the solution of (2.1) with stepsize $h$ linearly interpolated between steps. Thus

$$
\begin{gathered}
\Psi_{h}(t)=(1-\lambda) \Psi_{h}(\tau)+\lambda \Psi_{h}(\tau+h), \\
\tau \leq t<\tau+h, \lambda=t-\tau / h .
\end{gathered}
$$

Lemma 5.1. Let $\phi(t)$ be a weak solution of (3.1) for $t \in\left[t_{0}, t_{1}\right)$. Suppose $\phi(t) \notin D_{2}$ for $t \in\left(t_{0}, t_{1}\right)$ and $\phi\left(t_{0}\right) \in \mathrm{D}_{22} \cup D_{3}$. Let $\Psi_{h}$ satisfy $(2.1)$ on $\left[\mathrm{t}_{0}, \mathrm{t}_{1}\right)$. Then

$\forall \epsilon>0, \exists h_{0}>0, \delta>0 ; 0<h<h_{0} \&|| \Psi_{h}\left(t_{0}\right)-\phi\left(t_{0}\right)||<\delta \Rightarrow \forall t \in\left[t_{0}, \mathrm{t}_{1}\right)|| \Psi_{h}(t)-\phi(t)||<\epsilon$

Proof. We begin with two simple estimates:

(1) $\exists K>0$ such that $\forall \tau_{2}>\tau_{1}>0, \forall h>0$

$$
|| \phi\left(\tau_{2}\right)-\Psi_{h}\left(\tau_{2}\right)|| \leq|| \phi\left(\tau_{1}\right)-\Psi_{h}\left(\tau_{1}\right)||+K\left(\tau_{2}-\tau_{1}\right)
$$

This follows from the boundedness of $d \phi / d t$ and $\mathrm{d} \Psi_{h} / d t$.

(2) $\Gamma(\phi(t))=\Gamma\left(\Psi_{h}(t)\right)=\Gamma_{0} \& \phi(t) \in S \backslash D_{2}$ for $t \in\left(\tau_{1}, \tau_{2}\right) \rightarrow$

$$
\forall \tau_{2}>\tau_{1}>0, \forall h>0, h \rightarrow 0|| \phi\left(\tau_{2}\right)-\Psi_{h}\left(\tau_{2}\right)|| \leq|| \phi\left(\tau_{1}\right)-\Psi_{h}\left(\tau_{1}\right)||
$$

This follows from theorems (2.1) and (3.2) on the solutions to (2.1) and (3.1) for constant $\Gamma$.

Since $\phi(t) \notin D 2$ for $t \in\left(\mathrm{t}_{0}, \mathrm{t}_{1}\right)$, we have $\Gamma(\phi(t))=$ constant $=\Gamma_{1}$ for $t \in\left(t_{0}, \mathrm{t}_{1}\right)$. Since $\phi\left(t_{0}\right) \notin$ $D_{22} \cup D_{3}, \exists \delta_{0}$ such that ||$x-\phi\left(t_{0}\right)||<\delta_{0} \Rightarrow x \notin \mathrm{D}_{22} \cup D_{3}$. Let $B=\left\{x||\left|x-\phi\left(t_{0}\right)\right| \mid<\delta_{0}\right\}$. Then $\exists \Gamma_{2}$ such that $B=B_{1} \cup B_{2}, B_{1} \cap B_{2} \neq \varnothing$,

with

$B_{i}=\left(\left\{x \mid \Gamma(x)=\Gamma_{\mathrm{i}}\right\} \cap B \quad i=1,2\right.$.

Now define

$\lambda_{\tau}(x)=\min \left\{t-\tau \mid t>\tau, \phi(\tau)=x, \phi(t) \in D_{2}\right\}$

$\lambda^{\prime}(x)=\min \left\{t-\tau \mid t>\tau, \Psi_{h}(\tau)=x, \Psi_{n}(t) \in D_{2}\right\}$

Clearly $\lambda_{\tau}$ and $\lambda^{\prime}$ are continuous on $B$. Now choose $\delta_{1}, \delta_{2}<\delta_{0}$ such that 


$$
\begin{gathered}
|| x-\phi\left(t_{0}\right) \|<\delta_{1} \& x \in B_{1} \Rightarrow\left|\lambda_{t_{0}}(x)-\lambda_{t_{0}}\left(\phi\left(t_{0}\right)\right)\right|<\frac{\epsilon}{8 K} \\
|| x-\phi\left(t_{0}\right) \|<\delta_{2} \& x \in B_{2} \Rightarrow\left|\lambda_{t_{0}}(x)\right|<\frac{\epsilon}{8 K}
\end{gathered}
$$

From theorems (2.1) and (3.2), we can also find $\mathrm{h}_{0}>0$ such that

$\forall \tau \in\left(t_{0}, t_{1}\right) 0<h<h_{0} \& x \in B\left|\hat{\lambda}_{\tau}(x)-\lambda_{\tau}(x)\right|<\epsilon / 8 K$

Now consider the following two possibilities.

Case 1. $\Psi_{h}\left(t_{0}\right) \in B_{1}$. Then

$$
\left|\hat{\lambda}_{t_{0}}(x)-\lambda_{t_{0}}\left(\phi\left(t_{0}\right)\right)\right| \leq\left|\hat{\lambda}_{t_{0}}(x)-\lambda_{t_{0}}(x)\right|+\left|\lambda_{t_{0}}(x)-\lambda_{t_{0}}\left(\phi\left(t_{0}\right)\right)\right|<\frac{\epsilon}{4 K}
$$

Then $\Gamma\left(\Psi_{h}(t)\right)=\Gamma_{1}$ for a time interval of size $\geq\left(t_{1}-t_{0}\right)-\epsilon / 4 K$.

Case 2. $\Psi_{h}\left(t_{0}\right) \in B_{2}$. Then

$$
\left|\hat{\lambda}_{t_{0}}(x)\right| \leq\left|\hat{\lambda}_{t_{0}}(x)-\lambda_{t_{0}}(x)\right|+\left|\lambda_{t_{0}}(x)\right|<\frac{\epsilon}{4 K} .
$$

Now applying the second case, we find that $\Gamma\left(\Psi_{h}(t)\right)=\Gamma_{1}$ for an interval of size $\geq\left(t_{1}-t_{0}\right)-\epsilon / 2 K$. Now set $\delta=\min \left\{\epsilon / 2, \delta_{0}, \delta_{1}, \delta_{2}\right\}$. Applying equations (5.1) and (5.2) together with the preceding analysis, we find that

$\forall t \in\left[t_{0}, t_{1}\right)\left\|\phi(t)-\Psi_{h}(t)\right\| \leq\left\|\phi\left(t_{0}\right)\right\|-\Psi_{h}\left(t_{0}\right) \|+\mathrm{K} \cdot \epsilon / 2 \mathrm{~K}<\epsilon / 2+\epsilon / 2=\epsilon$.

This is the required result.

Theorem 5.2. Let $\phi(t)$ be a weak solution of (3.1) for $t \epsilon\left[t_{0}, t_{1}\right)$. Suppose $\phi(t) \notin D_{22} \cup D_{3}$ for $t \epsilon$ $\left[t_{0}, t_{1}\right)$ and that $\phi(t) \in D_{2}$ for finitely many $t$. Let $\Psi_{h}$ satisfy $(2.1)$ on $\left[t_{0}, t_{1}\right)$ and suppose $\Psi_{h}\left(t_{0}\right)=$ $\phi\left(t_{0}\right)$. Then

$\forall \epsilon>0, \exists h>0, \forall t \epsilon\left[t_{0}, t_{1}\right)\left\|\Psi_{h}(t)-\phi(t)\right\|<\epsilon$.

Proof. Fix $\epsilon>0$. Let the intersections of $\phi(t)$ with $D_{2}$ occur at $\tau_{1}<\ldots<\tau_{n}$. Let $\tau_{0}=t_{0}, \tau_{n+1}=t_{1}, \delta_{n+1}$ $=\in$. Using the previous lemma, we can successively choose $\left(\delta_{n}, h_{n}\right), \ldots,\left(\delta_{0}, h_{0}\right)$ to ensure that $0<\mathrm{h}<\mathrm{h} \&|| \psi(\tau)-\phi(\tau)||<\delta \Rightarrow \forall \mathrm{t} \varepsilon\left[\tau, \tau_{\mathrm{t}}\right)|| \psi(\mathrm{t})-\phi(\mathrm{t})||<\delta$

Choosing $h=\min \left\{h_{0}, \ldots, h_{n}\right\}$ and noting that $\left\|\psi_{h}\left(t_{0}\right)-\phi\left(t_{0}\right)\right\|=0<\delta_{0}$, we obtain the theorem. In removing the time dependence we begin with the vector field (3.1) and set $\mathrm{t}^{\prime}=\ln \mathrm{t}-\ln t_{0}$ (which is well defined for $t \geq t_{0}>0$ ). Then

$$
d x / d t=d x / d t / d t^{\prime} / d t=\Gamma(x)-x
$$

This equation is autonomous and simpler to work with. Conveniently, in the new time variable, the solution is defined on $[0 . \infty)$.

Results about the original system can easily be recovered by setting $t=t_{o} e \mathrm{t}^{\prime}$. 
Theorem 5.3 Let $G=(N, \hat{S}, \hat{P})$ be given. Let $G^{*}=\left(N, \hat{S}, \hat{P}^{*}\right)$ where

$$
\hat{P}^{(i)^{*}}=\hat{P}^{(i)}+C^{(i)}, i=1, \ldots, N
$$

and $C^{(i)}: \hat{S}^{(1)} \times \ldots \times \hat{S}^{(N)} \rightarrow \mathfrak{R}$ is independent of the i-th component. Then $G$ and $G^{*}$ have the same continuous fictitious play dynamics (5.3).

Proof. Clearly, (5.3) depends on $\mathrm{P}^{\wedge}$ through $\Gamma(x)$, and $\Gamma^{(i)}(x)$ depends on $\mathrm{P}^{\wedge}$ only through differences of the form $E_{\alpha}^{(i)}(x)-E_{j}^{(i)}(x)$. Since $C^{(i)}$ is independent of the i-th component, the theorem follows.

\section{SPECIFIC FICTITIOUS PLAY DYNAMICS: EXAMPLE}

The discontinuous vector field (5.3) defined by the continuous FP dynamics is characterised by a division of phase space into finitely many regions within which the dynamics is a trivial flow to a sink. Each $x \in S$ can be associated with its optimal strategy $\Gamma(x)$. This provides a useful way of following dynamics. Let

$$
L=\left\{\left(\alpha_{1}, \ldots, \alpha_{N}\right)\left[1 \leq \alpha_{i} \leq n_{i}\right\}\right.
$$

We have the labelling map

$$
\begin{gathered}
R: S \rightarrow L . \\
x \rightarrow\left(\alpha_{1}, \ldots, \alpha_{N}\right) \text { where } \Gamma^{(i)}(x)=e_{\alpha_{i}}^{(i)}
\end{gathered}
$$

Thus $S$ is divided into regions $R^{-1}(\alpha)$ which share the same label (and have the same optimal strategy). We can associate a (possibly infinite) string of symbols in $L$ to a solution $\phi(t)$ of (5.3) by listing the successive regions traversed by $\phi(t)$.

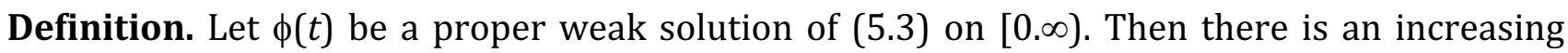
sequence $\left\{\tau_{i}\right\}^{n_{i=1}},=0$ (possibly infinite) such that:

(1) $R\left(\phi\left(\tau_{i}\right)\right) \neq R\left(\phi\left(\tau_{i+1}\right)\right)$ for $1 \leq i \leq n-1 . \varepsilon$

(2) $\tau_{i}<t<\tau_{i+1} \Rightarrow R(\phi(t))=R\left(\phi\left(\tau_{i}\right)\right)$ or $R(\phi(t))=R\left(\phi\left(\tau_{i+1}\right)\right)$.

(3) If $n$ is finite, $t>\tau_{n} \Rightarrow R(\phi(t))=R\left(\phi\left(\tau_{n}\right)\right)$.

The specific dynamics for $\phi$ is given by

$$
\gamma: \phi \rightarrow R\left(\phi\left(\tau_{1}\right)\right) R\left(\phi\left(\tau_{2}\right)\right) \ldots \epsilon L^{\prime} .
$$

We write $[\gamma(\phi)]_{n}$ for the $\mathrm{n}$-th symbol in the sequence $\gamma(\phi)$. The specific dynamics is clearly independent of the choice of sequence $\left\{\tau_{i}\right\}$ satisfying the conditions above.

Suppose $x(t) \in S \backslash D_{2}$. Let $R(x(t))=\alpha$. Then the next intersection of $x(t)$ with $D_{2}$ will occur at (1 $\lambda) x+\lambda \Gamma(x))$ for the smallest $\lambda \in(0,1]$ such that

$$
E i, \alpha_{1} \neq \alpha_{i} E_{\alpha_{1}}^{(i)}((1-\lambda) x+\lambda \Gamma(x))=E_{\alpha_{1}}^{(i)}((1-\lambda) x+\lambda \Gamma(x)) \text {. }
$$

We thus obtain the next intersection map $I$ :

$I:(x, t) \rightarrow((1-\lambda) x+\lambda \Gamma(x), t+\tau)$, where $\lambda=1-e^{-\tau}$.

This map can be extended to $x \in D_{2} \backslash\left(Z \cup D_{22}\right)$ by the uniqueness theorem. 


\section{Example}

Let $G$ be a two-player game with $n_{1}=n_{2}=2$. By theorem 5.3, we can add a constant row to $P^{(1)}$ and a constant column to $P^{(2)}$, to put the game in the standard form:

$$
P^{(1)}=\left(\begin{array}{ll}
A & B \\
0 & 0
\end{array}\right), \quad P^{(2)}=\left(\begin{array}{ll}
C & 0 \\
D & 0
\end{array}\right)
$$

Taking $A, B, C, D \neq 0$ to avoid degeneracies, we see that if $\operatorname{sgn}(A)=\operatorname{sgn}(B)$, the first player has a dominated strategy and if $\operatorname{sgn}(C)=\operatorname{sgn}(D)$, the second player has a dominated strategy. In either case, the dynamics is trivial. Without loss of generality, we can take

$A>0>B$. We see that $D^{(1)}=\left\{x \mid x_{1}^{(2)}=B / B-A=\mathrm{X}^{-}\right\}$and $D^{(2)}=\left\{x \mid x_{1}^{(1)}=D / D-C=\mathrm{Y}^{-}\right\}$.

Case 1. If $C>0>D$, then there are two stable equilibria at $((1,0),(1,0))$ and $((0,1),(0,1))$. The basics of attraction for these two equilibria are separated by the stable manifold of the unstable equilibria at $\left(\left(\mathrm{X}^{\prime}, 1-\bar{X}^{\prime}\right),\left(\mathrm{Y}^{\prime}, 1-\mathrm{Y}^{\prime}\right)\right)$ which takes the form of two line segments.

Case 2. Let us use the coordinates $(X, Y)$ defined by $X=\left(X^{(1)}, X^{(2)}\right)=\left(\left(\bar{X}^{\prime}+X,\left(1-\bar{X}^{\prime}\right)-X\right),\left(Y^{\prime}+Y,\left(1-Y^{\prime}\right)-Y\right)\right)$.

Writing the corresponding symbolic dynamics on the left, we have the following intersection maps:

$(2,1) \rightarrow(2,2),(X, 0) \rightarrow\left(0, X\left(1-Y^{\prime}\right) / X+\bar{X}^{\prime}\right)$

$(2,2) \rightarrow(1,2),(0, Y) \rightarrow\left(-Y X^{\prime} / Y+Y^{\prime}, 0\right)$,

$(1,2) \rightarrow(1,1),(X, 0) \rightarrow\left(0, X Y^{\prime} /-X+\left(1-\bar{X}^{\prime}\right)\right)$,

$(1,1) \rightarrow(2,1),(0, Y) \rightarrow\left(-Y\left(1-\bar{X}^{\prime}\right) /-Y+\left(1-Y^{\prime}\right), 0\right)$.

If we take $X>0, Y>0$ the symbolic dynamics for the corresponding trajectory is an infinite repetition of the fundamental cycle $(2,1)(2,2)(1,2)(1,1)$. By composing the four next intersection maps, we obtain the overall map around the fundamental cycle:

$T: A \rightarrow A$ where $\left.A=\{(X, 0)] 0<X \leq\left(1-\mathrm{X}^{\prime}\right)\right\}$.

$(X, 0) \rightarrow\left(c X /\left(1-Y^{\prime} X+c\right), 0\right)$ where $c=X^{\prime}\left(1-X^{\prime}\right) Y^{\prime}\left(1-Y^{\prime}\right)$.

We can find the n-fold iterate of $T$ :

$T^{n}:(X, 0) \rightarrow(c X / n(1-Y) X+c, 0)$

The time $\tau$ taken for a transition satisfies $\lambda=1-e(\exp -\tau)$. Hence, for a series of transitions with convex combination factors $\lambda_{1}, \ldots, \lambda_{n}$, the total transition, time $\tau$ satisfies:

$$
e^{-\tau}=e^{-\left(\tau_{1}+\ldots+\tau_{2}\right)}=e^{-\tau_{n}}=\left(1-\lambda_{1}\right) \ldots\left(1-\lambda_{n}\right) .
$$

Applying this relation (which still holds for an arbitrary number of players $N$ ), the time $\tau$ taken to traverse the fundamental cycle turns out to satisfy:

$e(\exp -\tau)=c /\left(1-Y^{\prime}\right)\left(1-2 X^{\prime} Y^{\prime}\right) X+c$

So there are no periodic orbits for $2 \times 2$ games, but it is possible for trajectories to spiral in on the Nash equilibrium $\left(X^{\prime}, Y^{\prime}\right)$ as $t \rightarrow \infty$. 


\section{CONCLUSIONS}

In economics, it has become standard practice to assume that each agent anticipates the other agents' optimizing behaviour at all meta-levels. This is known as rational expectations. Each agent optimizes under the assumption that the other agents are optimizing under the assumption each agent is optimizing ... ad infinitum. To avoid this regress, we may consider models with bounded rationality. In these models, the players are equipped with a set of relatively simple "habits of play". The habit of play in the discrete fictitious play model is local greediness: at each play of the repeated game, each player simply chooses his optimal strategy relative to the observed strategy used for the other players. Habits of play often simplify the strategy space of iterated games by pruning them to Markov strategies with limited memory or to average value strategies that depend only on the observed probabilities of play.

Each habit of play induces a map from the set of (finite) games to the set of maps or flows defined on a suitable strategy space. We have argued that insights into games can be obtained by embedding them in dynamical systems: different facets of game will be revealed in different dynamical contexts. For instance, Smale (1980b) works with a system very similar to discrete fictitious play. Related approaches to bounded rationality using dynamic algebraic systems, i.e. automata, have been described by Canning (1988), Gottinger (1990), Rubinstein (1986).

In Smale's framework each player has access to the average payoffs of all of the players, and he gives simple criteria for a habit of play to guarantee for a player almost the $(C, C)$ payoff in Prisoner's Dilemma (essentially just play C(D) if the player is doing better (worse) than the other player). Fictitious play exposes the limitations of narrowly "rational" thinking in Prisoner's Dilemma; the approaches of Smale and Kreps et al. (1982) emphasize the possibility of cooperation emerging through appropriate signalling of intentions. In a sense, the game itself is indeterminate; it requires a dynamical setting to be completely defined.

A simple example would be the current budgetary game involving many government departments where managers devise 'greedy' rules on the basis of (common) knowledge of average allocations to other departments in repeated negotiated rounds.

\section{ACKNOWLEDGEMENT}

This is to appreciate very helpful comments earlier by D. Arkin and R. Selten on some technical results.

\section{Footnote}

We are mostly concerned with a restricted situation of the following type. We are given finitely many players labelled $1, \ldots N$. Each player chooses a strategy $S^{(1)}$ from a finite collection of strategies $\hat{S}^{(i)}$ which need not be identical for the different players. The strategy choices are made completely independently; no communication is allowed (i.e. the game is non cooperative). The i-th player is then rewarded with a payoff $\hat{P}^{(i)}\left(S^{(1)}, \ldots, S^{(N)} \in \mathfrak{R}\right.$. Each player attempts to maximise his own payoff $\hat{P}^{(i)}$.

\section{References}

Binmore, K.G., "Remodelled Rational Players”, STICERD Disc. Paper 87/149, London School of Economics, 1987.

Brown, G.W., "Iterative Solutions of Games by Fictitious Play" in Activity Analysis of Production and Allocation (T.C. Koopmans, Ed.) New York, Wiley, 1951.

Canning, David, "Rationality and Game Theory when Players are Turing Machines", STICERD Disc. Paper TE/88/183, London School of Economics, October 1988.

Fudenberg, D. and D. Kreps, “Learning Mixed Equilibria”, Games and Economic Behaviour, 5, 1993, 320 -367. 
Gaunersdorfer, A. and J. Hofbauer, "Fictitious Play" Shapely Polygons, and the Replicator Equation”, Games and Economic Behaviour 11, 1995, 279-303.

Gottinger, H.W., “Complexity of Games and Bounded Rationality”, Optimization Vol. 21, 1990, pp. 991-1003.

Hofbauer, J., “Stability for Best Response Dynamics”, Inst. For Mathematik, Univ. Wien, 1995, mimeo.

Kalai,E.,"Bounded Rationality and Strategic Complexity in Repeated Games“, Center for Math. Studies in Economics and Management Science, Northwestern Univ., Evanston, Illinois, Disc.Paper 783, June 1988

Kreps, D., P. Milgrom, J. Robert, and R. Wilson, “Rational Cooperation in the Finitely Repeated Prinsoner's Dilemma' Journal of Economic Theory 27, 1982, 245-252.

Kreps, D., M., Game Theory and Economic Modelling, Oxford University Press, Oxford, 1990.

Matsui, A., "Best Response Dynamics and Socially Stable Strategies”, Journal of Economic Theory, 57, 1992, 343362 .

Megiddo,N., Remarks on Bounded Rationality, IBM Almaden Research Center, Research Paper, San Jose, Ca. 1986

Metrick, A. and B. Polack, "Fictitious Play in 2x2 games: a geometric proof of convergence, Economic Theory 4, 1994, 923-933.

Monderer, D., D. Samet and A. Sela, "Belief Affirming in Learning Processes”, Israel Inst. of Business Research. Fac. of Management, Tel Aviv Univ., Working Paper No. 15/96, June 1996.

Robinson, Julia, “An Iterative Method of Solving a Game”, Annals of Mathematics, Vol. 53 (1951), pp. $296-301$.

Rosenmüller, J., “Über Periodizitätseigenschaften spieltheoretischer Lernprozesse”, Z. Wahrsch. verw. Gebiete 17, 1971, 259-308.

Rubinstein, A., "Finite Automata Play the Repeated Prisoner's Dilemma”, Journal of Economic Theory, Vol. 39 (1986), pp. 83-96.

Shapley, L.S., "Some Topics in Two Person Games”, in Dresher, M., Shapley, L.S. and Tucker, A.W., eds., Advances in Game Theory, Princeton University Press, Princeton, 1964.

Smale, Steve, “Differentiable Dynamic Systems”, in The Mathematics of Time, Springer, New York, 1980a.

Smale, Steve, “The Prisoner's Dilemma and Dynamical Systems Associated to Non-Cooperative Games", Econometrica, Vol. 48, 1980b,1617-1634. 